eISSN 2444-7986

DOI: https://doi.org/10.14201/orl.15900

Artículo de revisión

\title{
EXPLORACIÓN DE LA VÍA AÉREA EN EL PACIENTE PEDIÁTRICO
}

\section{Airway exploration in children}

\author{
Fernando GÓMEZ-SÁEZ1'; Ana I. NAVAZO-EGUÍA²; Cristina VEGA-VAL³; Esteban GÓMEZ- \\ SÁNCHEZ1 ; Alicia MIRÁS-VEIGA' ${ }^{1}$; Juan VALENCIA-RAMOS ${ }^{1}$
}

Hospital Universitario de Burgos. ${ }^{1}$ Unidad de Cuidados Intensivos Pediátricos Servicio de Pediatría. ${ }^{2}$ Servicio de Otorrinolaringología. ${ }^{3}$ Unidad de Neonatología. Servicio de Pediatría. Burgos. España.

Correspondencia: fgomezsa@saludcastillayleon.es

Fecha de recepción: 21 de marzo de 2017

Fecha de aceptación: 16 de mayo de 2017

Fecha de publicación: 19 de mayo de 2017

Fecha de publicación del fascículo: 1 de marzo de 2018

Conflicto de intereses: Los autores declaran no tener conflictos de intereses

Imágenes: Los autores declaran haber obtenido las imágenes con el permiso de los pacientes

Política de derechos y autoarchivo: se permite el autoarchivo de la versión post-print (SHERPA/RoMEO)

Licencia CC BY-NC-ND. Licencia Creative Commons Atribución-NoComercial-SinDerivar 4.0 Internacional

Universidad de Salamanca. Su comercialización está sujeta al permiso del editor

RESUMEN

Introducción y objetivo: El manejo de la vía aérea representa un reto constante en la práctica pediátrica. La broncoscopia se ha convertido en los últimos años en una técnica imprescindible en el diagnóstico y tratamiento de diversas anomalías del aparato respiratorio del niño. Las especiales características de la vía aérea pediátrica y la diferenciada patología que presenta otorgan a la broncoscopia pediátrica una entidad propia. La broncoscopia pediátrica es una técnica segura y con un gran número de aplicaciones, tanto diagnósticas como terapéuticas. La utilización de ambos tipos de broncoscopios (flexible y rígido) permite de aprovechar las ventajas de cada uno de ellos. La broncoscopia flexible en pediatría es un procedimiento relativamente sencillo y de bajo riesgo, que proporciona información anatómica y dinámica de las vías aéreas, además de estudios citológicos y microbiológicos. La sencillez y bajo riesgo de esta técnica, además de no precisar anestesia general permite su realización incluso, a la cabecera del paciente, lo que ha propiciado un campo cada vez más extenso de indicaciones. El propósito de este artículo es ofrecer una revisión sobre la actualidad del procedimiento de la broncoscopia pediátrica, especialmente en cuanto a sus indicaciones. Método: Revisión narrativa. Conclusión: La exploración endoscópica de la vía aérea es una técnica rentable en pediatría, sin apenas complicaciones y que puede ofrecer información diagnóstica muy valiosa, así como realizar determinados procedimientos terapéuticos. Es recomendable que todos los profesionales involucrados en el manejo de pacientes con patología de vía aérea conozcan sus indicaciones, contraindicaciones, complicaciones, así como sus aplicaciones terapéuticas.

PALABRAS CLAVE pediatría; vía aérea; broncoscopia; lavado broncoalveolar; broncoscopia intervencionista 

pediatric practice. In the last years, bronchoscopy has become an essential technique in the diagnosis and treatment of various abnormalities of the child's respiratory system. The special characteristics of the pediatric airway and the differentiated pathology it presents give pediatric bronchoscopy its own entity. Pediatric bronchoscopy is a safe technique with many applications, both diagnostic and therapeutic. The use of both types of bronchoscopes (flexible and rigid) allows to take advantage of each one of them. Flexible bronchoscopy in pediatrics is a relatively simple and low-risk procedure that provides anatomical and dynamic information on the airways, as well as cytological and microbiological studies. The simplicity and low risk of this technique, in addition to not requiring general anesthesia, allows it to be performed even at the head of the patient, which has led to an increasingly extensive field of indications. The purpose of this article is to provide a review on the timeliness of the pediatric bronchoscopy procedure, especially about its indications. Method: Narrative review. Conclusion: The endoscopic examination of the airway is a cost-effective technique in pediatrics, with little complications and can offer very valuable diagnostic information, as well as perform certain therapeutic procedures. It is recommended that all professionals involved in the management of patients with airway pathology should know their indications, contraindications, complications, as well as their therapeutic applications.

KEYWORDS

pediatrics; airway; bronchoscopy; bronchoscopy rigid; bronchoalveolar lavage; interventional bronchoscopy

\section{INTRODUCCIÓN}

La patología de vía aérea en pediatría es muy diversa y en ocasiones compleja, suponiendo un verdadero reto diagnóstico y terapéutico. El abordaje ideal de estos pacientes es multidisciplinar, ya que en la evaluación inicial, diagnóstico, tratamiento y seguimiento van a estar involucrados diferentes especialistas. Será de vital importancia una historia clínica y exploración física detalladas. La broncoscopia se ha convertido en los últimos años en una técnica imprescindible en el diagnóstico y tratamiento de diversas anomalías del aparato respiratorio del niño. Las especiales características de la vía aérea pediátrica y la diferenciada patología que presenta otorgan a la broncoscopia pediátrica una entidad propia. El desarrollo que ha experimentado la broncoscopia en los últimos años ha sido espectacular y muy especialmente en el campo de la pediatría. Tanto las aplicaciones diagnósticas de la broncoscopia como las terapéuticas han aumentado de forma considerable.

El propósito de este artículo es ofrecer una revisión sobre la actualidad del procedimiento de la broncoscopia, especialmente en cuanto a sus indicaciones, contraindicaciones y complicaciones.

\section{LA BRONCOSCOPIA EN NIÑOS}

La broncoscopia es un método diagnóstico y terapéutico que se emplea actualmente de forma habitual en la patología de vía aérea en los niños [1]. Los inicios históricos de la broncoscopia se remontan a finales del siglo XIX, cuando Gustav Killian empleó un tubo rígido para extraer un cuerpo extraño del bronquio de un adulto. Posteriormente a principios del siglo XX el laringólogo estadounidense Chevalier Jackson perfecciona la técnica y desarrolla nuevos accesorios. En los años setenta del siglo $X X$ se produjo un gran avance con la cuando Shigeto lkeda inventa y desarrolla el broncoscopio flexible de fibra óptica. No fue hasta 1978 cuando Robert E. Wood describe la aplicación del broncoscopio flexible en neumología pediátrica, por lo que ésta técnica es relativamente reciente en este grupo de edad [2]. Sin embargo, pese a su juventud, se trata de una técnica que ha experimentado un desarrollo espectacular en el campo de la pediatría, con un aumento considerable de aplicaciones diagnósticas y terapéuticas [3].

Existen algunas peculiaridades de la broncoscopia pediátrica respecto a la realizada en adultos, y en las que nos vamos a centrar a continuación [4]:

A. Diferencias anatómicas y funcionales con el adulto.

B. Material específico debido a las características de la vía aérea pediátrica.

C. Indicaciones diferentes: en el niño son más frecuentes las anomalías anatómicas y la aspiración de cuerpo extraño y son muy infrecuentes las neoplasias.

D. Necesidad de sedación adecuada para la realización de la técnica.

A. DiFERENCIAS ANATÓMICAS Y FUNCIONALES CON EL ADULTO

Existen diferencias importantes anatómicas, de tamaño, forma y posición entre la vía aérea 
del adulto y del niño. Estas diferencias serán más notables cuanto más pequeño sea el niño, y se limitarán a la diferencia de tamaño al final de la infancia y adolescencia $[5,6]$.

La vía aérea del infantil, con inmadurez de estructuras laringotraqueobronquiales, presenta mayor predisposición al colapso. La forma de la cabeza, con el occipucio prominente, hace que, en posición de decúbito supino, recomendada durante el sueño como factor protector ante el síndrome de muerte súbita del lactante, condicione una flexión cervical y cierre de la vía aérea. La relajación muscular durante el sueño también puede agravar esta tendencia al cierre de vía aérea (Figura 1).

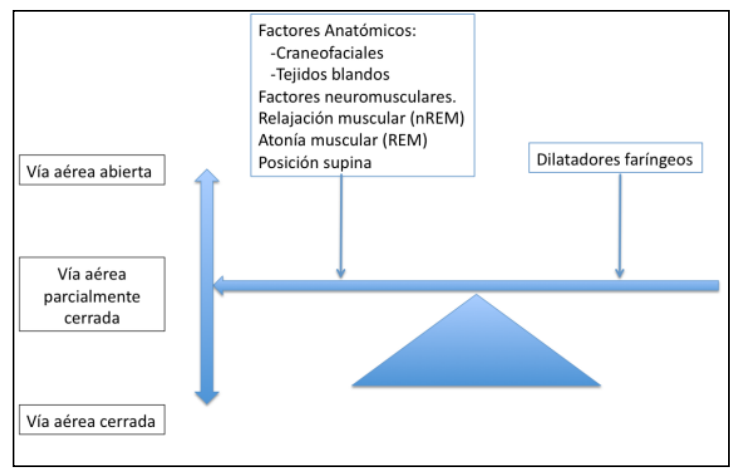

Figura 1. Fisiopatología en el cierre de la vía aérea en el paciente pediátrico.

Los neonatos, con respiración nasal, presentan narinas estrechas por lo que son propensos a la obstrucción a dicho nivel, pudiendo ocasionar dificultad respiratoria y de alimentación. La epiglotis, más larga y en forma de omega, se encuentra en una posición alta y anterior, cercana al paladar blando, lo cual favorece la respiración nasal durante la alimentación.

La lengua tiene un tamaño proporcionalmente mayor, ocupando completamente la cavidad oral.

La laringe se encuentra en el neonato a la altura de la primera vértebra cervical mientras que en el adulto a la altura de la quinta. En el menor de 8 a 10 años, la laringe tiene forma de cono truncado en cuya base se encuentra la zona más estrecha de la vía aérea infantil a nivel subglótico, a la altura del cartílago cricoides. En el adulto sin embargo la forma es cilíndrica, siendo las cuerdas vocales la porción más estrecha.
El crecimiento del tejido linfoide del anillo de Waldeyer (adenoides, amígdala lingual, amígdalas palatinas y tejido linfático faríngeo posterior) puede ocasionar obstrucción de la vía aérea del niño, especialmente desde el segundo año de vida hasta los 7 a 8 años de edad.

En cuanto al árbol traqueobronquial, la diferencia de calibre respecto al adulto, condiciona una mayor resistencia al paso del aire, favoreciendo que el flujo sea más turbulento incluso durante una respiración tranquila. Además del calibre más reducido, la mucosa respiratoria es más laxa y está más vascularizada, por lo que es más propensa a la inflamación durante su manipulación. Un mínimo grado de edema, el acúmulo de secreciones o broncoespasmo pueden aumentar de forma exponencial la resistencia al paso de aire, y puede conducir a una obstrucción de la vía aérea de evolución rápida (Figura 2).

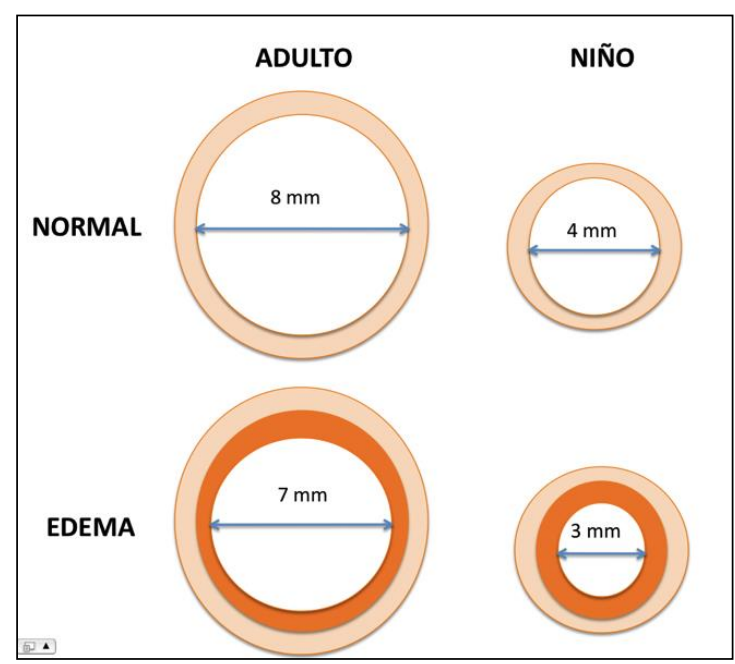

Figura 2. Corte transversal de la tráquea de un adulo y de un niño, en situación normal y con edema de $0,5 \mathrm{~mm}$. Este edema supone una disminución de la luz de la vía aérea de un $44 \%$ en el adulto, y de un $75 \%$ en el caso del niño. La resistencia al paso del aire también aumenta de forma crítica en el niño: en el adulto la resistencia se multiplica por 3, mientras que en este caso en el niño la resistencia se multiplica por 16 .

La pared torácica es más flexible, con las costillas horizontalizadas, por lo que la respiración y el mantenimiento de la capacidad pulmonar depende en gran medida de la musculatura respiratoria. El niño va a respirar con un menor volumen tidal y a mayor frecuencia. La menor capacidad residual funcional conlleva menor reserva en apnea y tendencia a hipoventila- 
ción. La musculatura diafragmática e intercostal del neonato y lactante es pobre en miofibrillas tipo I, que permiten los movimientos repetitivos, por lo que cualquier condición que conlleve un aumento del trabajo respiratorio puede conducirles a fatiga muscular y fracaso respiratorio. Existe además menor madurez del centro respiratorio, con menor respuesta a la hipercapnia y variaciones de $\mathrm{pH}$. En definitiva, la eficacia de la ventilación es menor en el niño, especialmente en el lactante pequeño y en el neonato, cuando la comparamos con el adulto. Por este motivo, en caso de apnea o ventilación alveolar inadecuada los niños presentan hipoxemia con mayor rapidez.

\section{B. MATERIAL ESPECÍFICO}

La broncoscopia en el niño aporta información anatómica y dinámica de la vía aérea. Va a permitir realizar estudios diagnósticos citológicos y microbiológicos y en ocasiones se puede emplear con objetivos terapéuticos [7].

En la actualidad se desarrollan dos técnicas, la broncoscopia rígida y la broncoscopia flexible, que no son excluyentes, sino complementarias.

\section{BRONCOSCOPIA RÍGIDA}

Los broncoscopios rígidos son tubos metálicos rectos de diferente longitud y grosor. Miden de 16 a $40 \mathrm{~cm}$ de longitud, con un diámetro interno que va desde 3,2 a $7,5 \mathrm{~mm}[1,8]$. Presentan el extremo distal biselado para no lesionar la glotis durante su inserción y varios orificios laterales en su tercio distal que van a permitir una ventilación colateral adecuada de los pacientes durante su uso. Disponen de un espacio central que consta de canal de trabajo y puertos de entrada para iluminación, lentes rígidas y aspiración.

Su inserción se realiza vía oral. Permite un control de la vía aérea y asegura una adecuada ventilación del paciente. Aporta una excelente calidad de visión, y gracias a su amplio canal de trabajo permite la utilización de una gran cantidad de pinzas y accesorios. Sin embargo, no permite una adecuada visualización de zonas distales de la vía aérea y para su utilización es necesaria la anestesia general del paciente en el quirófano.

Se trata de una técnica muy utilizada en broncoscopia infantil, ya que tiene un gran potencial terapéutico.

Sus principales indicaciones en pediatría son la extracción de cuerpos extraños en vía aérea, tratamiento de la obstrucción de vía aérea central y control de la hemoptisis masiva. Permite instrumentalización terapéutica de la vía aérea mediante el empleo de láser, colocación de endoprótesis, crioterapia, electrocoagulación, utilización de balones de dilatación, toma de biopsias, etc.

\section{BRONCOSCOPIA FLEXIBLE}

Los broncoscopios son instrumentos flexibles, de diferentes tamaños, que permiten su utilización en vía aérea, pudiendo llegar hasta regiones más distales que los broncoscopios rígidos [1, 3, 4, 9]. Los fibrobroncoscopios constan de haces de fibra óptica unidos entre sí para captar la imagen. Los videobroncoscopios, más modernos, no precisan del empleo de fibra óptica, y en su lugar disponen de un chip o cámara en el extremo distal que envía la imagen a un videoprocesador y a un monitor.

El broncoscopio flexible consta de tres partes: extremo proximal o cabezal, tubo flexible y extremo distal (Figura 3).

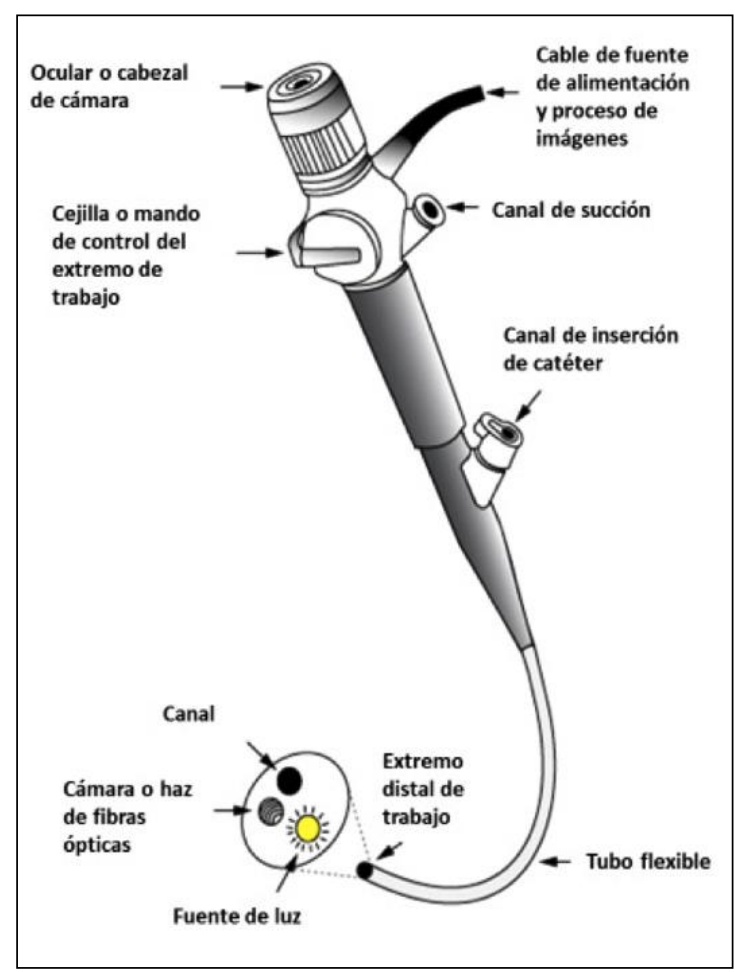

Figura 3. Esquema de broncoscopio flexible [10].

En el extremo proximal se encuentra el mando de control del extremo distal, la válvula de succión, el puerto de acceso al canal de trabajo y el cable de fuente de alimentación, fuente de 
luz y procesamiento de imágenes. En el caso de los fibrobroncoscopios se suele disponer de un ocular.

El cordón o tubo flexible puede ser de diferente longitud y diámetro.

El extremo distal tiene capacidad de flexión anterior $\left(120^{\circ}\right.$ a $\left.180^{\circ}\right)$ y posterior $\left(60^{\circ}\right.$ a $\left.130^{\circ}\right)$. En el extremo distal se encuentra el extremo del canal de trabajo, la fuente de luz y la cámara o el haz de fibras ópticas en función de si se trata de un fibrobroncoscopio o de un videobroncoscopio.

Los equipos se completan con una torre con videoprocesador, sistema de grabación, monitor y fuente de luz (Figura 4).
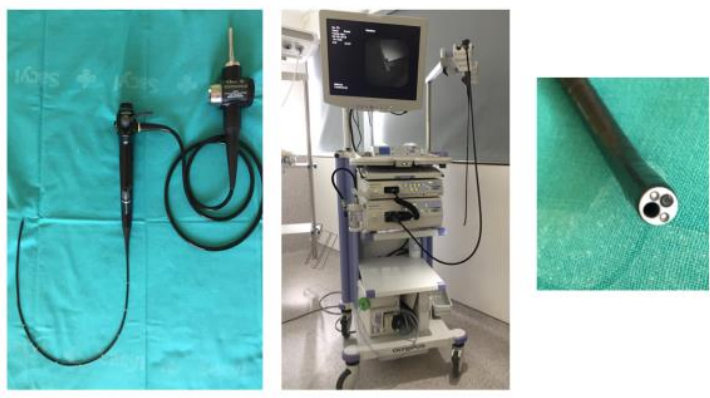

Figura 4. De izquierda a derecha: Imagen general de videobroncoscopio. Torre con videoprocesador, sistema de grabación, fuente de luz y monitor. Detalle de extremo distal de videobroncoscopio.

Existen diferentes tamaños de broncoscopios flexibles de uso pediátrico, que cubren desde el recién nacido pretérmino hasta el tamaño adulto. La mayor limitación de los de pequeño diámetro es que no existe canal de trabajo o bien es de un diámetro muy reducido (Tabla 1).

Tabla 1. Características principales de los broncoscopios pediátricos y edad de aplicación [1].

\begin{tabular}{|lllll|}
\hline $\begin{array}{l}\text { Diámetro } \\
\text { externo }\end{array}$ & $\begin{array}{l}\text { Canal de } \\
\text { trabajo }\end{array}$ & Accesorios & TET & Edad \\
\hline $4,9 \mathrm{~mm}$ & $2-2,2 \mathrm{~mm}$ & $\begin{array}{l}\text { Pinzas biopsia. } \\
\text { Catéteres } \mathrm{y} \\
\text { cepillos } \\
\text { protegidos }\end{array}$ & $>6,5 \mathrm{~mm}$ & $>20 \mathrm{~kg}$ peso \\
\hline $3,6 \mathrm{~mm}$ & $1,2 \mathrm{~mm}$ & $\begin{array}{l}\text { Pinzas biopsia. } \\
\text { Cepillos. }\end{array}$ & $>5 \mathrm{~mm}$ & $\begin{array}{l}\text {-Estándar } \\
\text { pediátrico } \\
- \text { RNT }\end{array}$ \\
\hline $2,8 \mathrm{~mm}$ & $1,2 \mathrm{~mm}$ & $\begin{array}{l}\text { Pinzas biopsia. } \\
\text { Cepillos. }\end{array}$ & $>3,5 \mathrm{~mm}$ & $\begin{array}{l}\text { RNT } \\
\text { Pretérmino }\end{array}$ \\
\hline $2,2 \mathrm{~mm}$ & No contiene & No & $>2,5 \mathrm{~mm}$ & Pretérmino \\
\hline & & & & \\
\hline
\end{tabular}

Los rinofibrolaringoscopios, con un funcionamiento y estructura similar a los broncoscopios, pero de menor longitud, se emplean para el estudio de la vía aérea superior [4]. Existen de fibra óptica y también videolaringoscopios. La mayor limitante, en los tamaños empleados en niños, es el pequeño calibre o la ausencia de canal de trabajo.

Las principales ventajas de la broncoscopia flexible son una menor invasión respecto a la rígida, por lo que se puede realizar bajo sedación con respiración espontánea. El broncoscopio se puede introducir vía oral, nasal o a través de traqueostomía o del tubo endotraqueal y permite una adecuada visualización de zonas distales de la vía aérea.

Como contrapunto, la broncoscopia flexible no asegura la vía aérea ni permite la ventilación del paciente. El menor canal de trabajo respecto al rígido permite el uso de menor variedad de accesorios e instrumentos. La calidad de imagen, a pesar del gran avance que han supuesto los videobroncoscopios continúa siendo superior con las ópticas rígidas.

\section{PROCEDIMIENTO DE BRONCOSCOPIA FLEXIBLE [1,} 3]

Previamente al inicio del procedimiento se informará al niño y su familia, solicitándose consentimiento informado. El niño debe estar monitorizado, bajo supervisión continua por un facultativo con experiencia en sedación y manejo de la vía aérea pediátrica. Debe existir una colaboración estrecha y comunicación entre el endoscopista y el médico encargado de la sedación.

El operador se ubica en la cabecera del paciente. Si el acceso es oral se coloca un mordedor o abrebocas, no necesario si el acceso es nasofaríngeo. Se elegirá el broncoscopio adecuado en función de la edad del niño y se lubrica su extremo distal. Se debe conectar el sistema de aspiración si se dispone del mismo. $\mathrm{Si}$ la introducción es nasal se procede al estudio de la anatomía faríngea y laríngea. El paso translaríngeo se realiza centrando el extremo del fibrobroncoscopio en la comisura anterior de las cuerdas vocales, para introducirlo a continuación, mediante una flexión posterior, aprovechando una respiración del paciente. Para facilitar el paso y prevenir el laringoespasmo se puede instilar lidocaína a través del canal de trabajo del broncoscopio. Se debe valorar la anatomía traqueal, el eje de la tráquea y su movimiento o colapso con la ventilación, visua- 
lización de la carina, posibles zonas de compresiones o desplazamientos, zonas pulsátiles, etc. Se debe observar la existencia de posibles anomalías anatómicas, el aspecto de la mucosa, así como las características de las secreciones, así como proceder a la toma de muestras que se crean oportunas. El estudio del árbol bronquial debe ser sistemático, completo y secuencial.

\section{INDICACIONES DE LA BRONCOSCOPIA FLEXIBLE EN PEDIATRÍA}

Las indicaciones para realizar una broncoscopia flexible son las siguientes (Tabla 2) [1-4, 7 , 11]:

1. Exploración de vía aérea por sintomatología respiratoria o anomalías radiológicas, no explicables con métodos no invasivos.

2. Toma de muestras para estudios citológicos y microbiológicos.

3. Aplicaciones terapéuticas.

Tabla 2: Indicaciones de broncoscopia flexible en el paciente pediátrico.

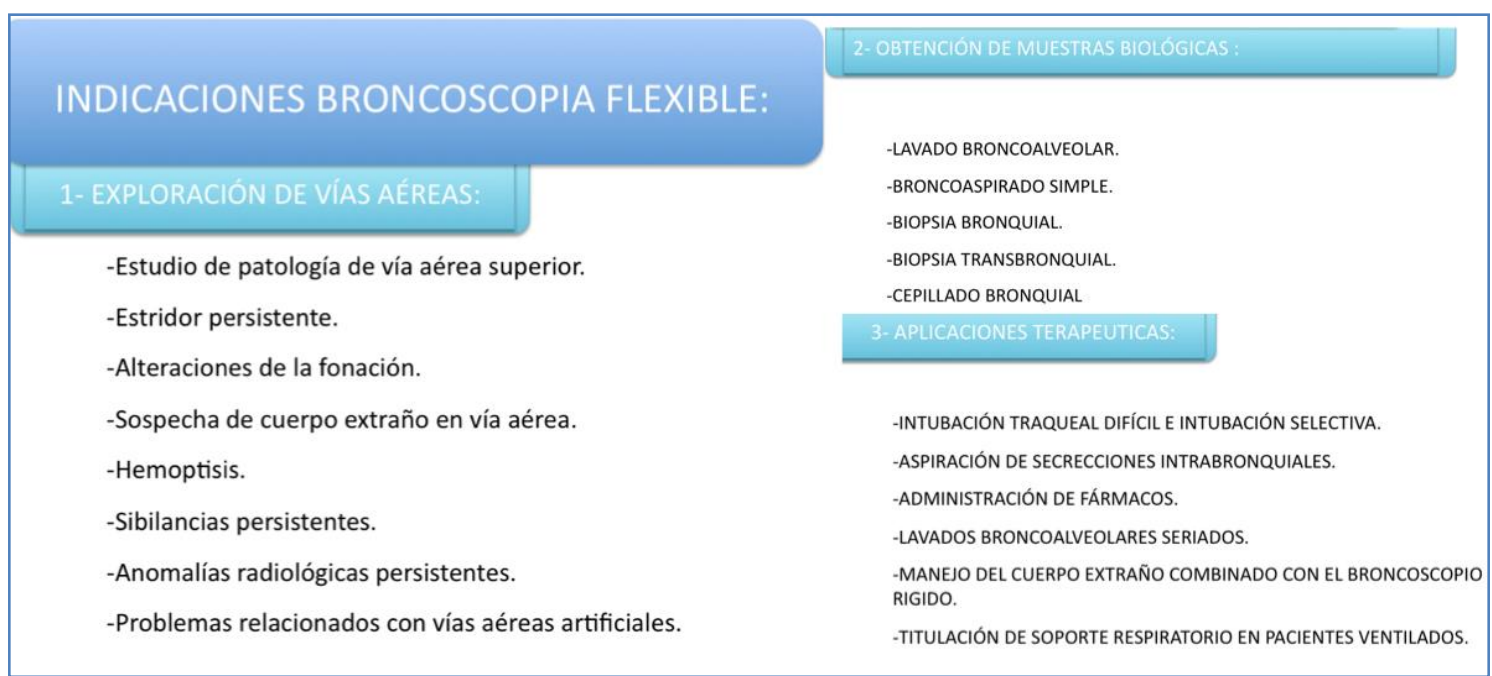

\section{EXPLORACIÓN DE LA VÍA AÉREA}

1.1. Estudio de patología de vía aérea superior [12].

La boncoscopia flexible puede contribuir al estudio diagnóstico del paciente con SAHS, valorando alteraciones nasofaríngeas como la hipertrofia adenoidea y amigdalar. Puede ser útil también para el estudio de patología nasal, de afecciones agudas del tracto respiratorio superior, sospecha de obstrucción u ocupación de vía aérea y en sospecha de alteración dinámica o funcional de la misma. Tiene un papel importante en el estudio de la disfagia y alteraciones de la deglución. En determinados síndromes y malformaciones craneofaciales puede ser de utilidad en la valoración de la vía aérea de estos pacientes.

1.2. Estridor persistente.

El estudio del estridor es una de las causas más frecuentes por las que se indica un estudio de vía aérea en edad pediátrica [13, 14]. Se define como un sonido respiratorio de tono variable producido por el paso de un flujo turbulento de aire en zonas de vía aérea con un calibre disminuido. Puede no existir en reposo y apreciarse con el llanto o el ejercicio. Puede ser agudo o crónico, congénito o adquirido. Dependiendo de la fase del ciclo respiratorio en la que es audible puede orientar hacia el nivel de la obstrucción: si es inspiratorio orienta a un problema supraglótico, mientras que el espiratorio es característico de vía aérea intratorácica. Si el estridor es bifásico, inspiratorio y espiratorio debemos pensar en patología glótica, subglótica o de tráquea extratorácica.

La causa más frecuente de estridor en la edad pediátrica es la laringomalacia [15]. Se trata de la anomalía congénita más frecuente de la vía aérea superior. Se presenta clásicamente como un estridor inspiratorio que se inicia en las primeras semanas de vida. No se conocen sus causas exactas, se cree puede deberse a un retraso en la maduración neuromuscular de 
las estructuras de soporte de la laringe, que ocasiona un colapso de las estructuras supraglóticas en inspiración. La mayoría de los niños con laringomalacia presentan una sintomatología leve que no suele requerir revisión endoscópica, con un curso benigno y autolimitado, con resolución del mismo a partir del primer año de vida. Sin embargo, en un 5-10\% de los casos la obstrucción de la vía aérea puede ser grave, condicionando dificultad para la alimentación, fallo de medro, apneas, cianosis e incluso puede conducir al desarrollo de hipertensión pulmonar y cor pulmonale. Es recomendable un estudio completo de la vía aérea en los pacientes con laringomalacia, ya que se están descrita la coexistencia de anomalías en vía aérea inferior en un $17 \%$ a $68 \%$ de los casos según diferentes series.

Ante un paciente que inicia clínica de estridor bifásico con antecedentes de intubación endotraqueal se debe pensar en la estenosis subglótica como posibilidad diagnóstica. Las congénitas suelen ser simétricas, mientras que las adquiridas, más frecuentes y secundarias a intubación, suelen ser asimétricas. La clínica será variable en función del grado de estenosis. El tratamiento puede ser conservador en formas leves, en casos moderados o graves puede ser preciso tratamiento endoscópico o quirúrgico, que se debe realizar en centros con experiencia en esta patología.

Es obligado el estudio endoscópico del paciente con estridor en los siguientes casos:

- Presentación atípica.

- Carácter bifásico.

- Persistencia prolongada en el tiempo.

- Crisis sofocación.

- Dificultad para alimentación. Estancamiento ponderal.

- Asociación con síndromes o malformaciones.

- Apneas o desaturaciones.

- Angustia familiar.

- Antecedentes de intubaciones prolongadas o repetidas.

\subsection{Alteraciones de la voz.}

La presencia de disfonía, afonía, Ilanto anómalo o cuadros que induzcan sospecha de patología laríngea son indicación de estudio endoscópico de la vía aérea. Esta sintomatología puede asociarse con estridor [1, 3].

La parálisis de cuerdas vocales es la segunda de las anomalías laríngeas más frecuentes en el recién nacido. Se produce una limitación a la abducción normal inspiratoria y puede ser unilateral o bilateral, congénita o adquirida.

La parálisis unilateral cursa con llanto débil, disfonía y puede cursar con dificultad para la alimentación. La mayoría de las parálisis de cuerdas vocales adquiridas son unilaterales del lado izquierdo, resultado de lesión traumática o compresión del nervio laríngeo recurrente izquierdo, ya que esta rama es más larga y vulnerable. El inicio de la clínica tras cirugía cardíaca debe hacer plantearse esta entidad. Se deben descartar otras patologías mediastínicas.

La parálisis bilateral cursa con estridor inspiratorio, llanto normal, o moderadamente afónico y puede ocasionar obstrucción de la vía aérea. Se asocia a alteraciones del sistema nervioso central como hipertensión intracraneal, síndrome de Arnold Chiari, hidrocefalia, etc.

El diagnóstico se realiza mediante endoscopia respiratoria con el paciente en ventilación espontánea. El pronóstico es mejor en las parálisis unilaterales y en las adquiridas. La evolución es favorable con recuperación de 2/3 de los casos en los primeros 6 meses. Las unilaterales no suelen requerir tratamiento, pues la laringe tiende a compensar con la cuerda sana. En las bilaterales la actitud será en principio expectante, siendo el manejo quirúrgico para garantizar la permeabilidad de vía aérea en casos de obstrucción grave y descompensaciones.

1.4. Sospecha de cuerpo extraño en vía aérea. Ante la sospecha de cuerpo extraño en vía aérea, la broncoscopia rígida presenta una considerable tasa de falsos negativos. Sin embargo, la broncoscopia flexible, que permite una exploración más completa y distal del árbol respiratorio tiene una alta sensibilidad, muy cercana al $100 \%$, en la detección de cuerpos extraños en vía aérea. Es por lo tanto muy útil como complemento al broncoscopio rígido en casos dudosos, para la confirmación o exclusión del diagnóstico 16. También para el control tras la extracción y descartar cuerpos extraños residuales.

Aunque existen publicaciones que avalan un buen rendimiento del broncoscopio flexible para la extracción de cuerpos extraños, el broncoscopio rígido es aún superior para este procedimiento, ya que asegura la vía aérea y permite mayor variedad de accesorios. El procedimiento ideal sería iniciarlo con el broncoscopio flexible, que permite una exploración de 
mayor alcance y la identificación del cuerpo extraño, proceder a la extracción con el broncoscopio rígido y por último efectuar una revisión final con el flexible.

\subsection{Hemoptisis.}

Se trata de un síntoma alarmante y poco frecuente en pediatría. En ausencia de explicación conocida requiere fibrobroncoscopia para localización del punto sangrante [17]. Se puede realizar aplicaciones terapéuticas como la instilación de adrenalina, sin embargo, para la hemoptisis masiva será de elección el broncoscopio rígido, ya que éste último, con un canal de trabajo de mayor tamaño permite la ventilación del paciente, así como aspiración e instrumentalización de forma simultánea.

Las causas más frecuentes de hemoptisis son problemas relacionados con vías aéreas artificiales, traqueostomías, hemorragia alveolar primaria, anomalías vasculares y patología endobronquial infecciosa o inflamatoria, siendo la patología tumoral excepcional.

\subsection{Sibilancias persistentes.}

Se recomienda la broncoscopia flexible en caso de sibilantes recurrentes o de difícil control con tratamiento médico, especialmente en niños pequeños, asociadas a asimetrías en auscultación y/o alteraciones radiológicas. Se deben descartar cuerpos extraños en vía aérea, así como anomalías estructurales o malácicas de la pared traqueobronquial $[2,17,18]$. La evaluación con broncoscopio flexible en pacientes con sibilancias inexplicables o persistentes evidencia alteraciones en más de la mitad de los casos, permitiendo además evaluar la presencia de datos sugerentes de reflujo gastroesofágico o de alteraciones de la deglución.

1.7. Anomalías radiológicas persistentes.

La presencia de atelectasias persistentes durante largos periodos sin mejoría con tratamiento médico y sin clara patología de base como fibrosis quística o asma, precisa de exploración con broncoscopio flexible para descartar factores anatómicos o presencia de cuerpo extraño. Se puede obtener además resultados terapéuticos con la aspiración de impactos mucosos.

La neumonía recurrente también es indicación de estudio endoscópico. La fibrobroncoscopia permite, además de descartar anomalías anatómicas o funcionales en la vía aérea, la realización de estudios diagnósticos citoquímicos y microbiológicos mediante broncoaspirado y lavado broncoalveolar.

En casos de hiperclaridad pulmonar localizada también está indicado el estudio endoscópico para descartar aspiración de cuerpo extraño, así como causas extrínsecas e intrínsecas de estenosis o disminución de calibre de vía aérea.

1.8. Problemas relacionados con vías aéreas artificiales.

La broncoscopia puede ser de utilidad para comprobar la permeabilidad y posición del tubo endotraqueal en pacientes intubados con soporte respiratorio con ventilación mecánica. También es fundamental para el diagnóstico de problemas que surjan durante la intubación y en pacientes en los que se produce fracaso de extubación. En estos casos, la exploración endoscópica puede descartar estenosis por intubación prolongada, lesiones secundarias a aspiración de secreciones, así como evaluación y limpieza de tapones mucosos en pacientes con o sin atelectasia radiológica.

Es fundamental también para el seguimiento y evaluación del paciente con traqueostomía, en los cuales la evaluación endoscópica permite medir la distancia desde el estoma hasta la carina para la elección de la cánula adecuada, así como el calibre de la misma previo a cambio de cánula o decanulación. También será de utilidad para la detección de complicaciones como estenosis o aparición de granulomas en vía aérea.

\section{OBTENCIÓN DE MUESTRAS BIOLÓGICAS PARA ESTUDIOS CITOLÓGICOS Y MICROBIOLÓGICOS.}

\subsection{Lavado broncoalveolar.}

Consiste en la instilación y posterior aspiración de cantidades estandarizadas de suero salino en uno o varios segmentos o subsegmentos pulmonares. La muestra obtenida aporta información de celularidad y bioquímica de la superficie epitelial del tracto respiratorio inferior, y se puede emplear para estudios microbiológicos $[1,3,4,19]$.

Como requisito, el broncoscopio debe disponer de canal de trabajo. Se debe realizar previamente una prueba de imagen, como radiografía de tórax o tomografía computarizada para delimitar el segmento idóneo donde realizarlo. Si existen zonas patológicas lo ideal es realizarlo en el segmento afecto, si la prueba de imagen es normal la rentabilidad diagnóstica será mayor en língula o lóbulo medio. El 
paciente debe tener una cifra de plaquetas superior a 50000/mmc y coagulación normal. Se debe realizar después de la inspección de la vía aérea y antes de procedimientos cruentos como cepillado o biopsia bronquial. Si el objetivo es la recogida de cultivos o pruebas microbiológicas, o ideal es realizar el lavado en primer lugar para disminuir el riesgo de contaminación.

El líquido empleado, que se instila y posteriormente se aspira por el canal de trabajo, es suero salino fisiológico, a temperatura ambiente o a $37^{\circ} \mathrm{C}$. Se realizará la instilación en varias alícuotas. En menores de $20 \mathrm{Kg}$ de peso se emplearán tres alícuotas, cada una con 3 $\mathrm{ml} / \mathrm{Kg}$. En pacientes con más de $20 \mathrm{Kg}$ de peso se emplearán alícuotas de $20 \mathrm{ml}$ hasta un máximo de $3 \mathrm{ml} / \mathrm{Kg}$. La aspiración se realiza empleando bajas presiones (de -25 a $-50 \mathrm{mmHg}$ ) para evitar el colapso de la vía aérea distal.

El lavado broncoalveolar tiene aplicaciones concretas en pediatría:

- Estudio de infecciones pulmonares en pacientes inmunocomprometidos. En pacientes inmunodeprimidos, oncológicos o trasplantados con disnea e hipoxemia con nuevos infiltrados pulmonares, debe realizarse antes de iniciar el tratamiento antibiótico y tras 48 horas de inicio de tratamiento si no ha habido mejoría. El rendimiento diagnóstico es superior al $80 \%$.

- Niños inmunocompetentes con neumonías graves que no responden de forma adecuada al tratamiento o precisan ventilación mecánica, y en neumonías nosocomiales en niños intubados y ventilados.

- Estudio de enfermedades pulmonares no infecciosas en las que es el método diagnóstico de elección: proteinosis alveolar, hemorragia alveolar, histiocitosis pulmonar y neumonía por acúmulo de lípidos.

- El lavado broncoalveolar puede ser útil en otras enfermedades como neumonía eosinofilica, neumonitis por hipersensibilidad, síndromes aspirativos, enfermedad pulmonar intersticial, sibilantes recurrentes graves, fibrosis quística.

- Tiene indicación terapéutica en el tratamiento de la proteinosis alveolar.

\subsection{Broncoaspirado simple.}

Consiste en la recogida y procesamiento de muestras recogidas por aspiración directa de secreciones de vías respiratorias mayor calibre, tras la instilación de pequeñas cantidades de suero salino fisiológico.

\subsection{Biopsia bronquial,}

La biopsia bronquial es la realizada con una pinza en una lesión endobronquial o en la mucosa $[1,3,19]$. Permite estudiar epitelio, membrana basal y en ocasiones musculo liso. Puede utilizarse para el estudio de enfermedades granulomatosas, tumores endobronquiales 0 de enfermedades fúngicas crónicas. También puede ser de utilidad en el estudio de la discinesia ciliar, pero la rentabilidad es similar en el cepillado nasal por lo que se prefiere esta última por su menor invasión.

\subsection{Biopsia transbronquial.}

Mediante el uso de una aguja que atraviesa la pared bronquial, permite obtener muestras de parénquima pulmonar para su posterior procesamiento, pudiendo evitar la realización de una toracotomía 1,3,19. Es de elección para el diagnóstico de los episodios de rechazo en los pacientes post trasplante pulmonar. Tiene menos valor para el diagnóstico de otras enfermedades como bronquiolitis obliterante o enfermedad pulmonar intersticial, donde la afectación puede ser parcheada y es preferible la biopsia abierta. Puede ser de utilidad en enfermedades granulomatosas como tuberculosis miliar y sarcoidosis. En el paciente inmunodeprimido puede ayudar a establecer el diagnóstico de infección por citomegalovirus.

Se recomienda prueba de imagen previa, así como control de hemograma y coagulación. Es recomendable su realización con el paciente intubado o con mascarilla laríngea, bajo anestesia general en el quirófano, ya que esto permite un mejor manejo en caso de sangrado. La utilización de fluoroscopia facilita su realización, para valorar la posición de la pinza. Pueden aparecer complicaciones como sangrado y neumotórax, siendo poco frecuentes.

\subsection{Cepillado bronquial.}

Esta técnica utiliza un cepillo que se introduce a través del canal de trabajo, realizándose un cepillado que puede utilizarse para el estudio de la mucosa bronquial o para obtener cultivos de las secreciones. Los broncoscopios de mayor tamaño permiten el uso de cepillos protegidos, recubiertos por un catéter, que evita la contaminación de la muestra en el canal de trabajo o en las vías respiratorias. Con los broncoscopios pediátricos con canal de trabajo de 
1,2 mm sólo puede utilizarse cepillos no protegidos.

Se trata de una técnica útil para el diagnóstico de tuberculosis, neumonía en pacientes con ventilación mecánica, infecciones pulmonares en inmunodeprimidos y discinesia ciliar.

\section{Aplicaciones TerapÉUticas}

3.1. Intubación endotraqueal difícil e intubación selectiva.

El broncoscopio flexible puede actuar como guía para la intubación en casos de vía aérea difícil, en pacientes con anomalías craneofaciales, síndromes polimalformativos, politraumatizados con inmovilización de columna cervical, así como cuando se requiera intubación selectiva bronquial $[1,3,20]$.

\subsection{Aspiración de secreciones.}

La aspiración de secreciones, impactos mucosos o coágulos pueden mejorar la situación respiratoria de algunos pacientes, y contribuir a la resolución de atelectasias que pueden motivar dificultad para la ventilación o extubación del paciente. La tasa de expansión radiológica, no obstante, no supera el $50 \%$ en la mayoría de las series. El principal limitante en la edad pediátrica es el pequeño diámetro del canal de trabajo del broncoscopio $(1,2 \mathrm{~mm}$ de canal de trabajo en los broncoscopios de $2,8 \mathrm{~mm}$ y de $3,6 \mathrm{~mm}$ ), con mayor tendencia al colapso y obstrucción que los modelos de adultos.

\subsection{Administración de fármacos.}

Se puede utilizar el broncoscopio para la administración de fármacos a través del canal de trabajo. Está descrito su uso para administración de mucolíticos, antifúngicos, surfactante, etc.

\subsection{Lavados broncoalveolares seriados.}

De elección en el tratamiento de la proteinosis alveolar.

\subsection{Cuerpo extraño en vía aérea.}

Aunque existen publicaciones que avalan el buen rendimiento de la broncoscopia flexible para la extracción de cuerpos extraños, la mayoría de los centros la consideran limitada a la localización del cuerpo extraño, la revisión tras el procedimiento para descartar la presencia de cuerpos extraños residuales y la extracción de cuerpo extraño en regiones distales donde no llega el broncoscopio rígido, empleándose de manera conjunta con este último (ver apartado 1.4).

3.6. Titulación de soporte respiratorio en pacientes ventilados.

En pacientes con patología respiratoria que precisan de ventilación no invasiva, la broncoscopia puede ser de utilidad para titular el soporte respiratorio óptimo que precisa el paciente, ya que aporta información dinámica del comportamiento de la vía aérea del paceiten ventilado, así como el grado de colapso con diferentes niveles de presión [1].

Contraindicaciones de la broncoscopia flexible en pediatría.

La indicación de una broncoscopia flexible es una decisión que debe ser valorada en función del cociente riesgo-beneficio de cada paciente. Existen contraindicaciones absolutas y relativas $[1,3]$ (Tabla 3).

Complicaciones de la broncoscopia flexible en pediatría.

La broncoscopia es un procedimiento generalmente seguro, pero no exento de complicaciones [1, 3] (Tabla 4). Las posibles complicaciones pueden ser derivadas de los factores de riesgo del paciente, del tipo de procedimiento, de una adecuada sedación, de una elección inadecuada del material y de la inexperiencia del broncoscopista.

Tabla 3: Contraindicaciones de la broncoscopia flexible.

ABSOLUTAS
-Hipoxemia refractaria grave.
-Inestabilidad hemodinámica.
-Diátesis hemorrágica no corregida (plaquetas< $20000 / \mathrm{mm}^{3}$ )
-No autorización por parte de paciente, padres o tutores.
RELATIVAS
-Cardiopatías congénitas cianosantes. Aumento circulación colateral.
-Hipertensión pulmonar grave.
-Alteraciones de la coagulación.
-Broncoespasmo.
-Inmunodeprimidos con disfunción respiratoria severa.

El traumatismo nasal y la epistaxis son complicaciones relativamente frecuentes, que se resuelven con compresión y en ocasiones con adrenalina tópica. 
La desaturación e hipoxemia pueden deberse a causas inherentes al procedimiento y al paciente, así como a déficit o exceso de sedación. El fibrobroncoscopio aumenta la resistencia en las vías aéreas, lo que junto a la sedación puede producir hipoxemia e hipercapnia. El lavado broncoalveolar y las maniobras de aspiración pueden producir colapso alveolar. Por estimulación vagal, se puede producir espasmo laríngeo y apneas. También por estímulo vagal o en niños con hiperreactividad bronquial se puede producir cierto grado de broncoespasmo. Estas complicaciones se pueden atenuar manteniendo al paciente en una situación cardiorrespiratoria estable, eligiendo el material de un tamaño adecuado, optimizando la sedación y analgesia, y llevando a cabo el procedimiento con rapidez.

Tabla 4: Complicaciones de la broncoscopia flexible.

MAYORES
-Desaturación < $90 \%$ e hipoxemia
-Apneas.
-Neumotórax.
-Hemorragia pulmonar
MENORES
-Traumatismo nasal y epistaxis.
-Tos y/o broncoespasmo. Laringoespasmo.
-Reacciones vagales.
-Vómitos. Naúseas.
-Fiebre.

El neumotórax es una complicación rara, puede ocurrir con la biopsia transbronquial. También puede producirse por un mal uso del broncoscopio, especialmente si hay movimientos bruscos, si se usa aire u oxígeno a presión por el canal de trabajo.

La hemorragia grave es excepcional en la broncoscopia infantil, pueden estar asociadas a lesiones endobronquiales muy vascularizadas, raras en niños, a lavado broncoalveolar en pacientes con alteración de la coagulación y especialmente la realización de biopsia transbronquial.
La aparición de fiebre tras el procedimiento se produce hasta en un $15 \%$ de los procedimientos, especialmente si se ha realizado lavado broncoalveolar. Se relaciona con liberación de citoquinas, o con bacteriemias en inmunodeprimidos.

\section{SEDACIÓN EN BRONCOSCOPIA FLEXIBLE PEDIÁTRICA}

La broncoscopia flexible es una técnica que puede generar en el niño ansiedad, disconfort y también dolor, por lo que va a precisar una sedación adecuada. Además, el paciente neonatal y pediátrico no es colaborador como un adulto, por lo que si la sedación no es óptima la prueba puede ser de muy dificultosa o incluso de imposible realización [1, 4, 21].

Existe la posibilidad de desencadenar potentes reflejos protectores de la vía aérea, que pueden dificultar la exploración, poner en riesgo la oxigenación y ventilación y provocar daño de estructuras anatómicas al paciente.

El procedimiento requiere información detallada a los padres y al niño, así como consentimiento informado. Se debe realizar una valoración preanestésica individualizada de cada paciente.

En el paciente ambulatorio se recomienda ayuno de 3 a 6 horas según la edad y alimentos que tome (menor tiempo en lactantes o neonatos con lactancia materna). La prueba se debe realizar en un entorno en el que se pueda desarrollar tanto endoscopia como sedación con seguridad, con material necesario para posibles complicaciones y personal entrenado en ambas técnicas. Antes del alta se requiere recuperación completa de consciencia y comprobación de tolerancia oral.

En el paciente hospitalizado la prueba se puede realizar en sala de endoscopia como el paciente ambulatorio, o bien en áreas de críticos. Se puede realizar sin soporte respiratorio, o bien con diferentes tipos como oxigenoterapia, ventilación no invasiva o ventilación mecánica convencional.

Los pacientes deben estar monitorizados durante la exploración, se recomienda al menos electrocardiograma, frecuencia cardíaca, frecuencia respiratoria, tensión arterial no invasiva y pulsioximetría continua. Es muy recomendable la capnografía.

El grado o profundidad de sedación se puede monitorizar con BIS (índice biespectral). En algunos pacientes, como aquellos con laringomalacia o traqueomalacia, donde un exceso de sedación puede sobreestimar el grado de 
colapso, esta monitorización va a permitir una sedación consciente, bien tolerada por el paciente y que puede simular la situación o comportamiento de su vía aérea durante el sueño natural.

Existe una amplia variedad de fármacos que pueden ser empleados para la sedación de estos pacientes (Tabla 5). La elección de los fármacos dependerá del grado de sedación deseado, de las características del paciente y de la experiencia del personal que realiza la sedación.

Tabla 5. Fármacos empleados en sedación para broncoscopia pediátrica.

\begin{tabular}{l} 
SEDANTES - HIPNÓTICOS: \\
\hline -INHALADOS: Sevoflurano, Óxido nitroso \\
-INTRAVENOSOS: Midazolam, Propofol, Dexmetomidina, Ketamina \\
\hline ANALGÉSICOS-OPIOIDES: \\
\hline -Fentanilo, Remifentanilo, Morfina. \\
\hline FÁRMACOS COADYUVANTES: \\
\hline -Atropina \\
-Lidocaína. \\
-Dexametasona. \\
-Analgésicos no opioides. \\
-Relajantes musculares.
\end{tabular}

\section{CONCLUSIONES}

La broncoscopia o exploración endoscópica de la vía aérea es una técnica rentable en pediatría, sin apenas complicaciones y que puede ofrecer información diagnóstica muy valiosa, así como realizar determinados procedimientos terapéuticos.

Es recomendable que todos los profesionales involucrados en el manejo de pacientes con patología de vía aérea conozcan sus indicaciones, contraindicaciones, complicaciones, así como sus aplicaciones terapéuticas.

\section{BIBLIOGRAFÍA}

1. Pérez Frías FJ, Pérez Ruiz E, Caro Aguilera P. Broncoscopia pediátrica y técnicas asociadas. $1^{\mathrm{a}} \mathrm{ed}$. Ergon. 2014. ISBN 978-8415950-97-4.

2. Wood RE, FinkR. Applications of flexible fiberoptic bronchoscopes in infants and children. Chest. 1978;73: 737.
3. Pérez Frías J, Moreno Galdó A, Pérez Ruiz E, Barrio Gómez de Agüero Ml, Escribano Montaner A, Caro Aguilera P. Normativa SEPAR. Normativa de broncoscopia pediátrica. Arch Bronconeumol. 2011; 47:350-60.

4. Zafra Anta MA, Luna Paredes MC. Endoscopia de la vía aérea. Pediatr Integral 2016; 20(2): 128e1-128.e8.

5. Dickison AE. The normal and abnormal pediatric upper airway. Recognition and managementof obstruction. Clin Chest Med 1987;583-596.

6. Garrido Galindo C, Flores Hernández SS, Nuñez-Pérez Redondo C. Diferencias anatomofuncionales y endoscópicas entre la vía aérea del niño y la del adulto. Rev Inst Nal Enf Resp Mex. 2007; 20(2): 142-148.

7. Wood RE. The emerging role of flexible bronchoscopy in pediatrics. Clin Chest Med 2001;22:311-7.

8. Díaz-Agero P, Canseco F, Gil JL. Indicaciones y técnica de la broncoscopia rígida. Broncoscopia diagnóstica y terapéutica. Madrid; Ergon; 2007.p 53-70.

9. Wood RE, Postma D. Endoscopy of the airway in infants and children. L Pediatr. 1988;112:1-6.

10. Islam S, Mathur PN, Finlay G. Flexible bronchoscopy in adults: preparation, procedure technique, and complications. UpToDate 2016.

11. Midulla F, De Blic J, Barbato a, Bush A, Ceber $\mathrm{E}$, et al. Flexible endoscopy of paediatric airways. Eur Respir J.2003;22:698-708.

12. FiguerolaMulet $\mathrm{J}$, Osona Rodriguez de Torres B, Llull FerretjansM, Román Piñana JM. Contribución de la fibrobroncoscopia al diagnóstico de las enfermedades de la vía aérea superior. An Pediatr.2005;63:137-42.

13. Wood RE. Evaluation of the upper airway in children. Curr Opin Pediatr.2008;20:266-71. 
14. Boudewyns A, Claes J, Van de Heyning P. Clinical practice: an approach to stridor in infants and children. Eur J Pediatr.2010;169:135-41.

15. Valencia Ramos J, Mirás Veiga A, Alonso Álvarez ML, Gómez Sáez F, Oyágüez Ugidos PP, del Blanco Gómez I, et al. Conocimiento de los pediatras sobre la laringomalacia: ¿siempre es un proceso banal? Rev Pediatr Aten Primaria. 2016;18:e63-e72.

16. Pérez Frías J, Caro Aguilera P, Pérez Ruiz E, Moreno Requena L. Tratamiento del cuerpo extraño intrabronquial. Broncoscopia combinada en neumologíaa infantil. An Pediatr. 2010;72:67-71.

17. Cakir E, Ersu RH, Uyan ZS, et al. Flexible bronchoscopy as a valuable tool of persistent wheezing in children. Int $\mathrm{J}$ Pediatric Otorhinol.2009;73:1666-8.
18. Weinberger M, Abu-Hasan M. Pseudoasthma: when cough, wheezing and dypsnea are not asthma. Pediatrics. 2007;120:855-64.

19. Escribano MontanerA, Moreno Galdo A. Grupo de técnicas de la Sociedad Española de Neumología Pediátrica. Técnicas fibrobroncoscópicas especiales: lavado broncoalveolar, biopsia bronquial y biopsia transbronquial. An Pediatr (Barc). 2005;62:352-66.

20. Fiadjoe J, Stricker P. Peddiatric difficult airway Management: current devices and thecniques. Anesthesiology Clin. 2009;5:191-5.

21. De Blic J, Telion C. Sedation and anasthesia for bronchsocopy. En: Priftis KN, Anthracopoulos MB, Eber E, Koumbourlis AC, Wood RE, eds. PAediatric bronchoscopy. Basel: Karger; 2010.p.22-9. 